\title{
A PRIORI INFORMATION IN IMAGE SEGMENTATION: ENERGY FUNCTIONAL BASED ON SHAPE STATISTICAL MODEL AND IMAGE INFORMATION
}

\author{
Xavier Bresson, Pierre Vandergheynst and Jean-Philippe Thiran \\ Signal Processing Institute (ITS), \\ Swiss Federal Institute of Technology of Lausanne (EPFL) \\ $\mathrm{CH}-1015$ Lausanne, Switzerland \\ \{Xavier.Bresson, Pierre.Vandergheynst, JP.Thiran\}@epfl.ch
}

\begin{abstract}
In this paper, we propose an energy functional to segment objects whose global shape is a priori known thanks to a statistical model. Our work aims at extending the variational approach of Chen et al. [1] by integrating the statistical shape model of Leventon et al. [2]. The proposed energy functional allows us to capture an object that exhibits high image gradients and a shape compatible with the statistical model which best fits the segmented object. The minimization of the functional provides a system of coupled equations whose steady-state solution is the solution of the segmentation problem. Results are presented on synthetic and medical images.
\end{abstract}

\section{INTRODUCTION}

In image analysis, the image segmentation problem is a fundamental issue. Its aim is to find a partition of an image into a finite number of semantically important regions. From a variational analysis point of view, two main approaches are considered to perform the segmentation: the Mumford-Shah approach and the geodesic active contours approach [3]. During the last decade, the Geodesic Active Contours (GACs) method has been employed in many situations to detect objects such as medical structures. However, in some situations, the classical GACs are not appropiate. In particular, even though the GACs model is powerful enough to segment all kind of objects lying in images, it fails to segment an object whose shape is a priori known.

Some authors have proposed to incorporate prior shape knowledge into the GACs. Leventon, Grimson and Faugeras [2] have developed GACs employing a statistical shape model defined by a Principal Component Analysis (PCA) over the Signed Distance Functions (SDFs) of training shapes of the object to be segmented. In their approach, the active surface, which is represented by a level set function, evolves locally, based on image gradients and curvature and globally towards the maximum a posteriori position and shape of the object. In [1], Chen et al. have defined an energy functional which is minimized when the GACs have captured high image gradients and a shape prior of the object to be segmented. The shape prior is computed by averaging a training set of rigidly registered curves. Other authors have proposed models to integrate prior shape knowledge into the GACs model $[4,5,6]$. In this paper, we propose to extend the Chen et al.'s approach [1] by integrating the statistical model of shape proposed by Leventon et al. [2] in the energy functional. This functional is minimized when the GACs have captured high image gradients and a shape belonging to the statistical model of the object to be segmented.

In Section 2, we propose to briefly review some state of the art results. In Section 3, we define the new energy functional and derive the system of equations which minimizes the functional. In section 4 , we give information about the numerical implementation of the proposed segmentation model. Finally, in Section 5, we introduce the results on 2D synthetic and medical images.

\section{STATE OF THE ART}

\subsection{Geodesic Active Contours and Level Set Representation}

The first variational model which detected boundaries in images was done by Kass et al. [7]. The basic idea of their model is to locate sharp variations of the image intensity by minimizing an energy functional. Then, Caselles et al. [3] have proposed a new intrinsic energy functional $J_{2}(C)=\int_{0}^{1} g(|\nabla I(C(p))|)\left|C^{\prime}(p)\right| d p$ where the function $g$ is the edge-stopping function. $J_{2}$ is a new length, obtained by weighting the Euclidean element of length $d s$ by the function $g(|\nabla I(C(p))|)$ which contains information regarding the objects' boundaries. Caselles et al. have proved in [3] that the final curve is a geodesic in a Riemannian space. This geodesic is computed by the calculus of variations providing the Euler-Lagrange equations of $J_{2}$ and the gradient descent method which gives the flow minimizing the functional $J_{2}: \partial_{t} C=(\kappa g-$ $\langle\nabla g, \mathcal{N}\rangle) \mathcal{N}$ where $\mathcal{N}$ is the unit normal to the curve $C$ and $\kappa$ its curvature. Finally, objects in images are found out when the steady-state solution of the previous equation is achieved.

To deal with changes of topology during the curve evolution, the level set method proposed by Osher and Sethian [8] is essential. The main idea is to embed the curve $C$ as the zero level set of a higher dimensional function $\phi: \mathbb{R}_{+} \times \Omega \rightarrow \mathbb{R}$. Then, a curve's evolution $\partial_{t} C=F \mathcal{N}$ can be re-written in a level set formulation: $\partial_{t} \phi=F|\nabla \phi|$. The evolution of the curve $C$ coincides with the motion of the zero level set of the function $\phi$.

\subsection{Statistical Shape Model}

In order to model a geometric shape and incorporate it into a GACs' framework, we think that the shape model of Leventon et al. [2] is quite suitable. Indeed, first of all, it is basically a level set-based model since it is built from a set of level set functions. Secondly, the shape model is computed by the PCA on the SDFs of curves which provide a stronger tolerance than the parametric curves to slight misalignments during the alignment process of the training 
data since the values of neighboring pixels are highly correlated in SDFs. From a geometric point of view, the PCA analysis determines the best orthonormal basis $\left\{\mathbf{e}_{1} \ldots \mathbf{e}_{m}\right\}$ of $\mathcal{R}^{m}$ to represent a set of $n$ points $\left\{\phi_{1} \ldots \phi_{n}\right\}$ in the sense of the least squares fitting. In our work, a SDF $\phi_{j}$ is represented by $N^{q}$ samples ( $q$ is the number of dimensions and $N$ the number of samples for each dimension), hence the space $E$ is $\mathcal{R}^{N^{q}}$. In the PCA, a point $\phi_{j}$ is represented by the formula: $\phi_{j}=\bar{\phi}+\sum_{i=1}^{m} \alpha_{j i} \mathbf{e}_{i}+R_{j}=\hat{\phi}_{j}+R_{j}$ where $\bar{\phi}$ is the geometric average of $\left\{\phi_{j}\right\}_{j}$ and $R_{j}$ is the distance between the point $\phi_{j}$ and $\hat{\phi}_{j}$, the orthogonal projection of the vector $\phi_{j}$ into the space spans by the basis $\left\{\mathbf{e}_{i}\right\}_{i}$.

The PCA aims at finding out the vectors $\left\{\mathbf{e}_{i}\right\}_{i}$ which minimize the quadratic error $\varepsilon=\sum_{j=1}^{n}\left\|R_{j}\right\|^{2}$ under the constraint that these vectors form an orthonormal basis in $\mathcal{R}^{m}$. These vectors are given by the eigenvectors of the covariance matrix $\boldsymbol{\Sigma}=\frac{1}{n} M M^{\top}$ where $M$ is a matrix whose column vectors are the $n$ centered training SDF $\phi_{j}$. The vectors $\left\{\mathbf{e}_{i}\right\}_{i}$ correspond to the principal variation directions of the set of $n$ points. They are the principal components. Moreover, the first $p$ principal axes define a reduced $p$-dimensional vector space in $\mathcal{R}^{m}$ equivalent to an hyperplan minimizing the sum of squared distances between this hyperplan and the set of $n$ points. It is important to note that the fitting goodness of this $p$-D hyperplan in relation to the set of points can be measured in percentage by the formula $\beta=\sum_{k=1}^{p} \lambda_{k} / \sum_{k=1}^{n} \lambda_{k}$ where $\lambda_{k}$ are the eigenvalues of $\boldsymbol{\Sigma}$. It is possible to arbitrarly fix the fitting percentage $\beta$ and represent the data in a sub-vector space of dimension $p$. In practice, the importance of principal modes of a training set strongly decreases because the training data represent the same class of objects. Thus, only a small number of eigenmodes is useful for our purpose. These $p$ principal components are sorted in a matrix $\mathbf{W}_{p}$. Thus, the projected data $\hat{\phi}$ in the $p$-D space of a data $\phi$ in $\mathcal{R}^{m}$ is given by the following equations:

$$
\left\{\begin{array}{l}
\hat{\phi}=\mathbf{W}_{p} \mathbf{x}_{p c a}+\bar{\phi} \\
\mathbf{x}_{p c a}=\mathbf{W}_{p}^{\top}(\phi-\bar{\phi}) .
\end{array}\right.
$$

where $\mathbf{x}_{p c a}$ is the vector of eigencoefficients.

\section{NEW FUNCTIONAL FOR IMAGE SEGMENTATION}

\subsection{Variational Formulation of the Segmentation Model}

In this section, we formulate a new energy functional which is minimized once the GACs have captured high image gradients and formed a shape compatible with the statistical model of the object to be segmented. Our approach aims at extending the work of Chen et al. [1] by integrating the statistical shape model of Leventon et al. [2]. We propose the following energy functional:

$$
\begin{aligned}
F\left(C, \mathbf{x}_{T}, \mathbf{x}_{p c a}\right)=\int_{0}^{1}\left\{g_{e}(|\nabla I(C(p))|)+\right. \\
\left.\beta \hat{\phi}^{2}\left(g_{\mathbf{x}_{T}}(C(p)), \mathbf{x}_{p c a}\right)\right\}\left|C^{\prime}(p)\right| d p
\end{aligned}
$$

where $g_{e}$ is a decreasing function vanishing at infinity, $\hat{\phi}$ is a shape function provided by the PCA (1) over the training SDFs of the object to be segmented, $\mathbf{x}_{p c a}$ is the vector of PCA eigencoefficients, $g_{\mathbf{x}_{T}}$ is an element of a group of transformations and $\mathbf{x}_{T}$ is the vector of parameters. We note that $\mathbf{x}_{p c a}$ is independent of the spatial position of the shape function but the mean value $\bar{\phi}$ and the matrix of the first $p$ principal components $\mathbf{W}_{p}$ depend on the spatial location.
As in [1], we consider the rigid transformations (but the affine transformations can also be employed):

$$
\begin{aligned}
g_{\mathbf{x}_{T}}: \mathcal{R}^{2} & \rightarrow \mathcal{R}^{2} \\
x & \rightarrow g_{(\mu, \theta, T)}(x)=\mu R_{\theta} x+T,
\end{aligned}
$$

where the vector of rigid transformations $\mathbf{x}_{T}$ is composed of a scale parameter $\mu$, an angle of rotation $\theta$ and a vector of translations $T$.

We think that this new variational approach can segment an object in images whose global geometric shape is a priori known. Indeed, the first term of the functional $\int_{0}^{1} g_{e}\left|C^{\prime}\right| d p$ is the GACs classical functional which detects boundaries with the edge-stopping function $g_{e}$. The second term of the functional $\int_{0}^{1} \hat{\phi}^{2}\left|C^{\prime}\right| d p$ evaluates the similarity of the shape of the contour $C$ to that of the zero level set of $\hat{\phi}\left(\mathbf{x}_{T}, \mathbf{x}_{p c a}\right)$. Finally, $\beta$ is an arbitrary positive constant that balances the contributions of the boundary term and the shape term.

In the next section, we derive the set of coupled equations that minimize the functional (2) in the parametric (Lagrangian) and the implicit (Eulerian) formulations.

\subsection{Lagrangian and Eulerian Active Contours}

The energy functional (2) is minimized when the steady-state solution to a system of coupled equations are achieved. If the integrant of (2) is called $f$, the flow minimizing the functional $F$ w.r.t. the curve $C$ is the classical flow:

$$
\left\{\begin{array}{l}
\partial_{t} C(t, p)=(f \kappa-<\nabla f, \mathcal{N}>) \mathcal{N} \\
C(0, p)=C_{0}(p)
\end{array}\right.
$$

Then, the flows minimizing the functional $F$ w.r.t. the vector of rigid transformations $\mathbf{x}_{T}$ are:

$$
\begin{aligned}
& \left\{\begin{array}{l}
\partial_{t} \mathbf{x}_{T}(t)=-2 \beta \int_{0}^{1} \hat{\phi}<\nabla \hat{\phi}, \nabla_{\mathbf{x}_{T}} g_{\mathbf{x}_{T}}>\left|C^{\prime}\right| d p, \\
\mathbf{x}_{T}(0)=\mathbf{x}_{T 0},
\end{array}\right. \\
& \text { with } \quad \nabla_{\mathbf{x}_{T}} g_{\mathbf{x}_{T}}(C)=\left(\begin{array}{l}
\frac{\partial g_{\mathbf{x}_{T}}}{\partial \mu}(C)=R_{\theta} C \\
\frac{\partial g_{\mathbf{x}_{T}}}{\partial \theta}(C)=\mu \partial_{\theta} R_{\theta} C \\
\frac{\partial g_{\mathbf{x}_{T}}}{\partial T}(C)=\mathbf{1}
\end{array}\right) \text {. }
\end{aligned}
$$

The flows minimizing the functional $F$ w.r.t. the vector of eigencoefficients $\mathbf{x}_{p c a}$ are:

$$
\begin{array}{r}
\left\{\begin{array}{l}
\partial_{t} \mathbf{x}_{p c a}(t)=-2 \beta \int_{0}^{1} \hat{\phi} \nabla_{\mathbf{x}_{p c a}} \hat{\phi}\left|C^{\prime}\right| d p \\
\mathbf{x}_{p c a}(0)=\mathbf{x}_{p c a},
\end{array}\right. \\
\text { with } \quad \nabla_{\mathbf{x}_{p c a}} \hat{\phi}=\left(\begin{array}{l}
\mathbf{e}_{p c a}^{1} \\
\vdots \\
\mathbf{e}_{p c a}^{p}
\end{array}\right)
\end{array}
$$

and $\mathbf{e}_{p c a}^{i}$ the $i$ th principal components of the PCA model.

The steady-state solution of the system of equations (4), (5) and (7) provide the solution of the segmentation problem.

As we said in Section 2.1, the level set representation is essential to solve problems of interface propagation. In our work, we use the variational level set approach as presented in [9]. The level set formulations of the functional $F$ and the system of equations (4), (5) and (7) are:

$$
\begin{aligned}
F\left(\phi, \mathbf{x}_{T}, \mathbf{x}_{p c a}\right)= & \int_{\Omega}\left\{g_{e}(|\nabla I(x)|)+\right. \\
& \left.\beta \hat{\phi}^{2}\left(g_{\mathbf{x}_{T}}(x), \mathbf{x}_{p c a}\right)\right\} \delta(\phi)|\nabla \phi| d \Omega,
\end{aligned}
$$




$$
\left\{\begin{array}{l}
\partial_{t} \phi(t, x)=(f \kappa|\nabla \phi|-<\nabla f, \nabla \phi>) \delta(\phi) \\
\phi(t=0)=\phi_{0}\left(C_{0}\right)
\end{array}\right.
$$

where $\phi_{0}\left(C_{0}\right)$ is the SDF of the initial active contour $C_{0}$.

$$
\begin{gathered}
\left\{\begin{array}{l}
\partial_{t} \mathbf{x}_{T}(t)=-2 \beta \int_{\Omega} \hat{\phi}<\nabla \hat{\phi}, \nabla_{\mathbf{x}_{T}} g_{\mathbf{x}_{T}}>\delta(\phi)|\nabla \phi| d \Omega, \\
\mathbf{x}_{T}(0)=\mathbf{x}_{T 0},
\end{array}\right. \\
\text { with } \quad \nabla_{\mathbf{x}_{T}} g_{\mathbf{x}_{T}}(x)=\left(\begin{array}{l}
\frac{\partial g_{\mathbf{x}_{T}}}{\partial \mu}(x)=R_{\theta} x \\
\frac{\partial g_{\mathbf{x}_{T}}}{\partial \theta}(x)=\mu \partial_{\theta} R_{\theta} x \\
\frac{\partial \mathbf{g}_{T}}{\partial T}(x)=\mathbf{1}
\end{array}\right) .
\end{gathered}
$$

And for $i \in[1, p]$ :

$$
\left\{\begin{array}{l}
\partial_{t} \mathbf{x}_{p c a}^{i}(t)=-2 \beta \int_{\Omega} \hat{\phi} \mathbf{e}_{p c a}^{i} \delta(\phi)|\nabla \phi| d \Omega, \\
\mathbf{x}_{p c a}^{i}(0)=\mathbf{x}_{p c a}^{i},
\end{array}\right.
$$

\section{IMPLEMENTATION ISSUES}

Concerning the PCA model, we have aligned the training curves with the similarity measure of shapes given in [1]. We have used a fast algorithm [10] to compute the SDFs of the aligned training curves. Then, we have employed the C-code given by Numerical Recipies [11] to perform the singular values decomposition on the matrix $\boldsymbol{\Sigma}^{\text {dual }}=\frac{1}{n} M^{\top} M$ to extract the eigenvalues $\mathbf{e}_{\text {pca }}^{d, i}$ and the eigenvectors $\lambda_{\text {pca }}^{d, i}$. The PCA performed on $\boldsymbol{\Sigma}^{\text {dual }}$ rather than $\boldsymbol{\Sigma}$ gives fast and accurate results and the eigenvectors $\mathbf{e}_{p c a}^{i}$ and the eigenvalues $\lambda_{p c a}^{i}$ are given by $\mathbf{e}_{p c a}^{i}=M \mathbf{e}_{p c a}^{d, i}$ and $\lambda_{p c a}^{i}=\lambda_{p c a}^{d, i}$. Concerning the evolution equations (10), (11) and (13), they are numerically solved by iterating the following steps until convergence is reached:

1. Computation of $\hat{\phi}\left(\mathbf{x}_{T}, \mathbf{x}_{p c a}\right)$ by using (1) and by performing the rigid transformations (scaling, rotation and translations) with the polynomial B-splines interpolation method proposed by Unser [12]. With this technique, we can smoothly interpolate a function ( $\hat{\phi}$ in our case) from connected polynomial functions of a given degree (a degree 3 in our program).

2. Discretization of the hyperbolic terms $|\nabla \phi|$ and $\langle\nabla f, \nabla \phi>$ with the Engquist-Osher or the Godunov numerical flux function. Computations of the curvature and other spatial depending terms use standard central difference schemes. We have approximated the Dirac function $\delta(\phi)$ by a slightly regularized version as in [9].

3. Calculations of the left-hand side of (10), (11) and (13) are done with a forward difference approximation.

4. Redistancing the level set function every five iterations with the fast marching method of Adalsteinsson and Sethian [13].

\section{EXPERIMENTAL RESULTS}

\subsection{Synthetic Images}

We have tested our model in 2D synthetic images with a training set of ellipses. We have generated a set of 20 ellipses by changing the size of a principal axis with a Gaussian PDF. Then, we have applied the PCA on the training SDFs of ellipses and we have obtained one principal component that fits at $98.9 \%$ the set of 20 SDFs of ellipses. Figure 1 shows the shape functions $\hat{\phi}$ and the zero level sets corresponding to the mean and the eigenmode of variation of ellipses. In the first experiment on Figure 2, we have segmented an ellipse which is partially occluded by a vertical bar.

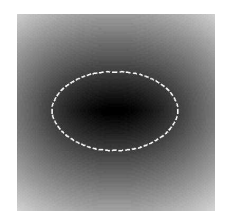

(a) $-1 \lambda_{1}$

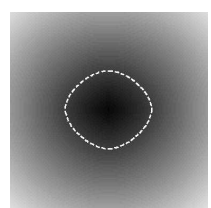

(b) Mean

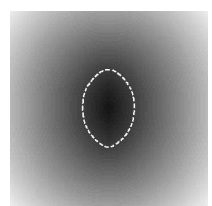

(c) $1 \lambda_{1}$
Fig. 1. Eigenmode of variation of ellipse SDFs.

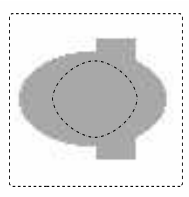

(a)

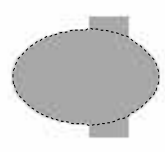

(b)

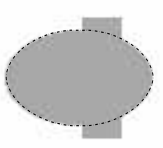

(c)
Fig. 2. (a) is an image with a binary ellipse occluded by a bar, the initial contour (the rectangle) and the initial zero level set of the shape model (the circle). (b) is the final active contour. (c) is the final zero level set of the shape model.

We can see that the active contour has captured high image gradients and a shape belonging to the statistical model which best fits the ellipse in the image. In the second experiment on Figure 3, we have captured an ellipse which is partially cut.

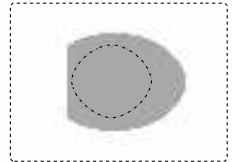

(a)

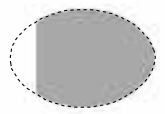

(b)

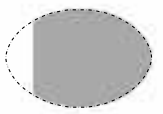

(c)
Fig. 3. (a) is an image with a binary ellipse partially cut, the initial contour (the rectangle) and the initial zero level set of the shape model (the circle). (b) is the final active contour. (c) is the final zero level set of the shape model.

\subsection{Medical Images}

We have also experimented our model in 2D medical images. We have used fifteen 2D images of left ventricles to build our statistical shape model. These fifteen 2D images are extracted from several slices of four T1-MRI images of healthy voluntaries. We have applied the PCA and we have obtained two principal components that fit at $88.2 \%$ the set of 15 SDFs of ventricles. Figure 4 shows the level set functions and the zero level sets corresponding to the mean and both eigenmodes of variation of the ventricle training set. The segmentation is presented on figure 5. We observe on Figure 5(c) that the active contour has well captured the left ventricle whereas the initial contour was around the two ventricles on Figure 5(a). This segmentation result could not be obtained without a shape prior with the same initial contour. The segmentation 


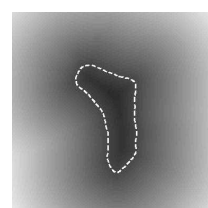

(a) $-2.5 \lambda_{1}$

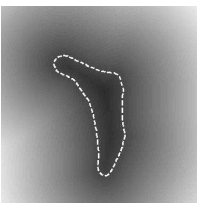

(d) $-2.5 \lambda_{2}$

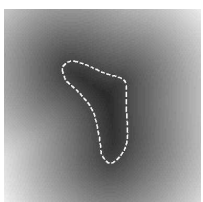

(b) Mean

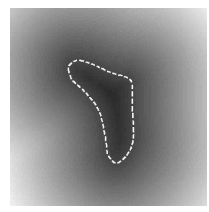

(e) Mean

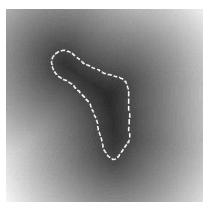

(c) $2.5 \lambda_{1}$

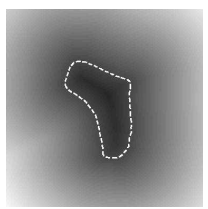

(f) $2.5 \lambda_{2}$
Fig. 4. Both eigenmodes of variation of left ventricles.

model has also provided on Figure 5(f) the shape of the statistical model which best fits the ventricle lying in the image.

\section{CONCLUSION AND FUTURE WORK}

In this paper, we have proposed a new variational model to solve the segmentation problem of an object whose global shape is a priori known through a statistical model. We saw that the active contours, which result to the minimization of the energy functional (2) is able to capture high image gradients and a shape of the statistical model which best fits the segmented object. Thus, this model simultenously takes into account local and global information in the segmentation process. This segmentation model can also be interpreted as a registration problem since we register a global shape to detect an object in the image.

Future works are focused on testing the model on new structures, performing the method on 3D images and using the affine transformations.

However, even if the model is less sensitive w.r.t. the initial condition thanks to the shape information, the initial condition has to be satisfactory to avoid bad local minima. Finally, the proposed model can capture only one object, which is limited since we lose the powerful property of the level set approach which can segment several objects simultaneously.

\section{REFERENCES}

[1] Y. Chen, H.D. Tagare, S. Thiruvenkadam, F. Huang, D. Wilson, K.S. Gopinath, R.W. Briggsand, and E.A. Geiser, "Using prior shapes in geometric active contours in a variational framework," IJCV, vol. 50(3), pp. 315-328, Dec 2002.

[2] M. Leventon, W. Grimson, and O. Faugeras, "Statistical shape influence in geodesic active contours," in Conf. of Computer Vision and Pattern Recognition, 2000, pp. 316323.

[3] V. Caselles, R. Kimmel, and G. Sapiro, "Geodesic active contours," IJCV, vol. 22(1), pp. 61-79, 1997.

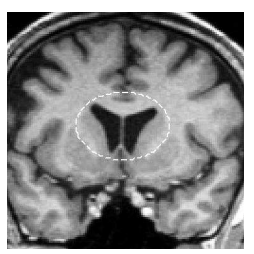

(a)

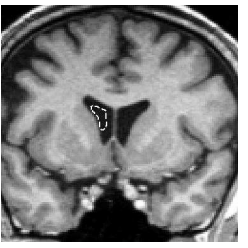

(d)

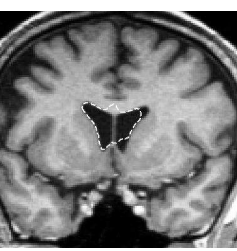

(b)

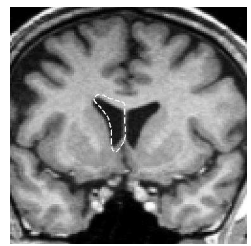

(e)

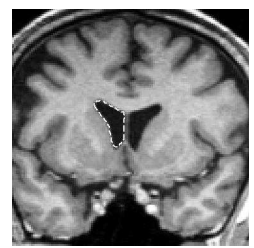

(c)

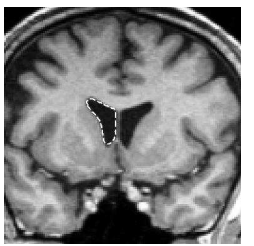

(f)
Fig. 5. (a) is the initial active contour, (b) is the active contour at a certain time, (c) is the final active contour, (d) is the initial zero level set of the shape model, (e) is the zero level set of $\hat{\phi}$ at the same time of (b) and (f) is the final zero level set of the shape model.

[4] A. Tsai, A. Yezzi, W. Wells, C. Tempany, D. Tucker, A. Fan, W.E. Grimson, and A. Willsky, "Model-based curve evolution techniques for image segmentation," in Conf. on Computer Vision and Pattern Recognition, 2001, pp. 463-468.

[5] D. Cremers, C. Schnrr, and J. Weickert, "Diffusion-snakes combining statistical shape knowledge and image information in a variational framework," in Workshop on Variational and Levelset Methods, July 2001, pp. 137-144.

[6] N. Paragios and M. Rousson, "Shape priors for level set representations," in Eur. Conf. in Computer Vision, June 2002.

[7] M. Kass, A. Witkin, and D. Terzopoulos, "Snakes: Active contour models," IJCV, pp. 321-331, 1987.

[8] S. Osher and J.A. Sethian, "Fronts propagating with curvature-dependent speed: Algorithms based on hamiltonjacobi formulations," J. Comp. Phys., vol. 79(1), no. 12-49, 1988.

[9] H. Zhao, T. Chan, B. Merriman, and S. Osher, "A variational level set approach to multiphase motion," Journal of Computational Physics, vol. 127, pp. 179-195, 1996.

[10] O. Cuisenaire and B. Macq, "Fast euclidean distance transformations by propagation using multiple neighbourhoods," Journal of CVIU, vol. 76(2), pp. 163-172, 1999.

[11] Numerical Recipies in $C++$ and $C$, Cambridge University Press.

[12] M. Unser, "Splines: A perfect fit for signal and image processing," IEEE Signal Processing Magazine, vol. 16(6), pp. 22-38, 1999.

[13] D. Adalsteinsson and J. Sethian, "A fast level set method for propagating interfaces," Journal of Computational Physics, vol. 118, pp. 269-277, 1995. 